\title{
A COMPLEX WHICH CANNOT BE PUSHED AROUND IN $E^{3}$
}

\author{
MICHAEL STARBIRD ${ }^{1}$
}

\begin{abstract}
This paper contains an example of a finite complex $C$ with triangulation $T$ which admits two linear embeddings $f$ and $g$ into $E^{3}$ so that although there is an isotopy of $E^{3}$ taking the embedding $f$ to $g$ there is no continuous family of linear embeddings of $C$ starting at $f$ and ending at $g$. No such example can exist in $E^{2}$.
\end{abstract}

1. Introduction. When considering complexes PL embedded in $E^{n}$, there are at least three types of motion of the complex which one could study. The most restrictive type would be motion in which each simplex is required to remain the same size and shape at each moment. Euler's flexible surface problem [4] is an example of a question concerning this type of motion. Less restrictive is the notion of a PL isotopy in which the motion involved is a continuous family of PL embeddings. In a PL isotopy $h_{t}(t \in[0,1])$, at every time $t$ there is a triangulation of the complex with respect to which $h_{t}$ is a linear embedding; however, there need not be one single triangulation with respect to which each of the $h_{t}$ 's is linear.

Between the two types of motion mentioned above is the linear isotopy with which this paper deals. A linear isotopy of a complex is the special kind of PL isotopy in which one triangulation of the complex does serve continuously for the entire family of embeddings. (See formal definition below.) One feature of linear isotopies different from PL isotopies is that any linear isotopy of a finite complex into $E^{n}$ can be extended to a linear isotopy of all of $E^{n}$ [2, Theorem 3.3]. One type of question associated with linear isotopies is when the existence of an ambient isotopy of $E^{n}$ which takes one linear embedding of a complex to another implies the existence of a linear isotopy of the complex between the same two embeddings.

Cairns proved [3] that if $f$ and $g$ are two linear embeddings of a finite complex $(C, T)$ into $E^{2}$ so that there is an orientation-preserving homeomorphism $h$ of $E^{2}$ with $h \circ f=g$, then there is a linear isotopy of $(C, T)$ taking $f$ to $g$. In this paper an example is presented to show that the analogous theorem for $E^{3}$ is false. In a future paper the author will prove a weakened version of Cairns' theorem in $E^{3}$, namely, where the linear isotopy in the

Received by the editors October 4, 1976.

AMS (MOS) subject classifications (1970). Primary 57A10, 57C99.

Key words and phrases. Linear isotopy, push.

${ }^{1}$ This work was supported by NSF grant MPS75-16158. 
conclusion of Cairns' theorem is with respect to some subdivision of $T$. (This theorem and questions related to the example are stated at the end of this paper.)

Definitions. Let $C$ be a complex with triangulation $T$. Then $h: C \rightarrow E^{n}$ is a linear embedding of $C$ (or $(C, T)$ ) into $E^{n}$ if. $h$ is an embedding which is linear on each simplex in $T$. A linear isotopy of $C$ (or $(C, T)$ ) is a continuous family of embeddings $h_{t}: C \rightarrow E^{n}(t \in[0,1])$ so that for every $t, h_{t}$ is a linear embedding of $C$ into $E^{n}$. A linear isotopy is called a simple push if it is fixed except on the star of one vertex. A push is a finite sequence of simple pushes performed one after the preceding.

2. A complex which cannot be pushed around. In [3], Cairns proved that if $f$ and $g$ are two linear embeddings of a finite complex $(C, T)$ into $E^{2}$ so that there is an orientation-preserving homeomorphism $h$ of $E^{2}$ with $h \circ f=g$, then there is a push of $(C, T)$ from $f$ to $g$. The example in this section shows that the situation is different in $E^{3}$.

The following observations about knots in $E^{3}$ are used in establishing the properties of the example.

LEMMA 2.1. Let $K$ be a $P L$ knot linearly embedded in $E^{3}$ so that for every $P L$ homeomorphism $h$ of $E^{3}, h(K)$ has at least as many edges as $K$ has. Let $v_{i}, v_{i+1}$, and $v_{i+2}$ be three consecutive vertices of $K$. Then $\operatorname{Int}\left(v_{i} v_{i+1} v_{i+2}\right) \cap K \neq \varnothing$.

Proof. Suppose no segment of $K$ intersects the interior of the 2-simplex $v_{i} v_{i+1} v_{i+2}$. If any edge $v_{j} v_{j+1}$ intersects the open segment $\left(v_{i} v_{i+2}\right)$, a slight adjustment of $v_{i}$ would cause $v_{j} v_{j+1}$ to miss the 2-simplex $v_{i} v_{i+1} v_{i+2}$ entirely. Therefore, we adjust $K$ so that no edge of $K$ intersects $\operatorname{Int}\left(v_{i} v_{i+1} v_{i+2}\right)$ nor intersects the open segment $\left(v_{i} v_{i+2}\right)$. Now the broken arc $v_{i} v_{i+1} \cup v_{i+1} v_{i+2}$ can be pushed to the segment $v_{i} v_{i+2}$ along the 2-simplex $v_{i} v_{i+1} v_{i+2}$ keeping the other edges of the adjusted $K$ fixed, thus embedding $K$ with fewer edges contrary to hypothesis.

Corollary 2.2. A simple closed curve $J$ in $E^{3}$ made up of five or fewer straight edges is unknotted.

Proof. If $J$ has 3 or 4 edges, it is not knotted. Let $J$ have vertices ordered consecutively as $v_{1}, v_{2}, v_{3}, v_{4}$, and $v_{5}$. We assume that the vertices are in general position. Let $P_{123}$ be the plane determined by $v_{1}, v_{2}$, and $v_{3}$. We assume without loss of generality that $v_{4}$ lies above $P_{123}$. By Lemma 2.1 , if $J$ is to be knotted, the 2-simplex $v_{1} v_{2} v_{3}$ must be pierced. Hence $v_{5}$ must lie below $P_{123}$. Then, however, $v_{2} v_{3} v_{4}$ is not pierced, showing that $J$ is not knotted.

EXAMPLE 2.1. This example is a 1-complex $(C, T)$ with two linear embeddings $f$ and $g$ of $C$ into $E^{3}$ so that there is an isotopy of $E^{3}$ taking $f(C)$ to $g(C)$ and yet for which there is no push nor linear isotopy $h_{t}(t \in[0,1])$ of $(C, T)$ with $h_{0}=f$ and $h_{1}=g$. 
The example is drawn in Figure 2.1. It has twelve vertices and seventeen edges.
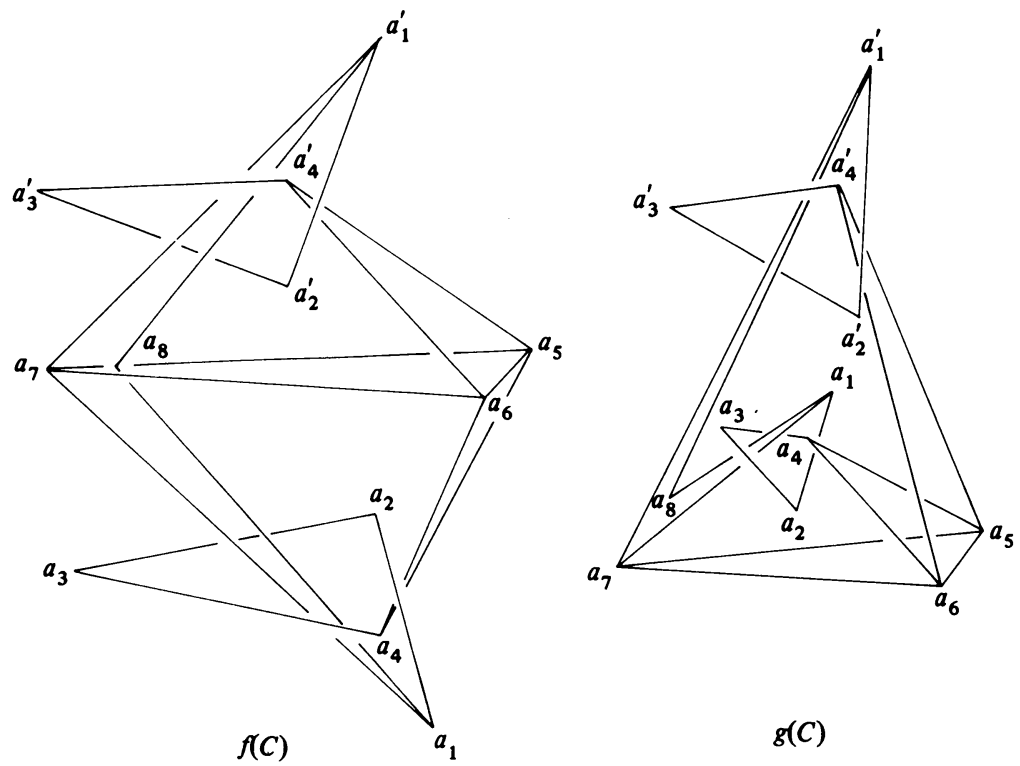

FIGURE 2.1

In order to establish the fact that this example has the properties claimed, we need to prove various facts about it.

First, we need to show that there is an isotopy of $E^{3}$ which moves $f(C)$ to $g(C)$. The isotopy is obtained by reaching down through triangle $a_{5} a_{6} a_{7}$ in $f(C)$ and pulling all the edges below $a_{5} a_{6} a_{7}$ through the triangle $a_{5} a_{6} a_{7}$ and arranging them as they should look in $g(C)$. Vertices $a_{1}^{\prime}, a_{2}^{\prime}, a_{3}^{\prime}, a_{4}^{\prime}, a_{5}, a_{6}$, and $a_{7}$ need not be moved in this process. Some edges will have to bend in intermediate stages of the isotopy, as we prove below. One method of demonstrating that this isotopy of $E^{3}$ exists is to build a model of $f(C)$ with string and then move it to $g(C)$ as described.

Next we show that no linear isotopy of $(C, T)$ starts at $f$ and ends at $g$.

Suppose there were a linear isotopy $h_{t}(t \in[0,1])$ of $(C, T)$ so that $h_{0}=f$ and $h_{1}=g$. Note that under $f$ the arc $a_{1}^{\prime} a_{8} a_{1}$ pierces the triangle $a_{5} a_{6} a_{7}$ whereas under $g$ it does not. Therefore there is an $s$ in $[0,1]$ so that $h_{s}\left(a_{1}\right)$ or $h_{s}\left(a_{1}^{\prime}\right)$ lies in the 2-simplex $h_{s}\left(a_{5}\right) h_{s}\left(a_{6}\right) h_{s}\left(a_{7}\right)$. We suppose that $h_{s}\left(a_{1}\right)$ does. (The other case is equivalent because of the symmetry of $C$.) The strategy of the proof is to prove that $h_{s}$ cannot be a linear embedding of the subcomplex of $C$ whose vertices do not have primes.

Let $P_{i j k}$ be the plane determined by $h_{s}\left(a_{i}\right), h_{s}\left(a_{j}\right)$, and $h_{s}\left(a_{k}\right)$.

The point $h_{s}\left(a_{4}\right)$ is either above, below, or on the plane $P_{567}$. It is not on $\boldsymbol{P}_{567}$ because no plane contains four vertices of a knotted simple closed curve with only six vertices such as $h_{s}\left(a_{1} a_{2} a_{3} a_{4} a_{5} a_{7}\right)$. Assume that $h_{s}\left(a_{4}\right)$ is below $P_{567}$. 
By Corollary 2.2 and Lemma 2.1, we know that the 2-simplex $h_{s}\left(a_{1}\right) h_{s}$ $\left(a_{5}\right) h_{s}\left(a_{7}\right)$ must be pierced by an edge of the knot $h_{s}\left(a_{1} a_{2} a_{3} a_{4} a_{5} a_{7}\right)$. The only edges available are $h_{s}\left(a_{2} a_{3}\right)$ and $h_{s}\left(a_{3} a_{4}\right)$. Assume that $h_{s}\left(a_{3} a_{4}\right)$ does. (See Figure 2.2.)

Similarly the 2-simplex $h_{s}\left(a_{1}\right) h_{s}\left(a_{6}\right) h_{s}\left(a_{7}\right)$ must be pierced by $h_{s}\left(a_{2} a_{3}\right)$ or $h_{s}\left(a_{3} a_{4}\right)$ since $h_{s}\left(a_{1} a_{2} a_{3} a_{4} a_{6} a_{7}\right)$ is a knot. Since it is not pierced by $h_{s}\left(a_{3} a_{4}\right)$, it must be pierced by $h_{s}\left(a_{2} a_{3}\right)$.

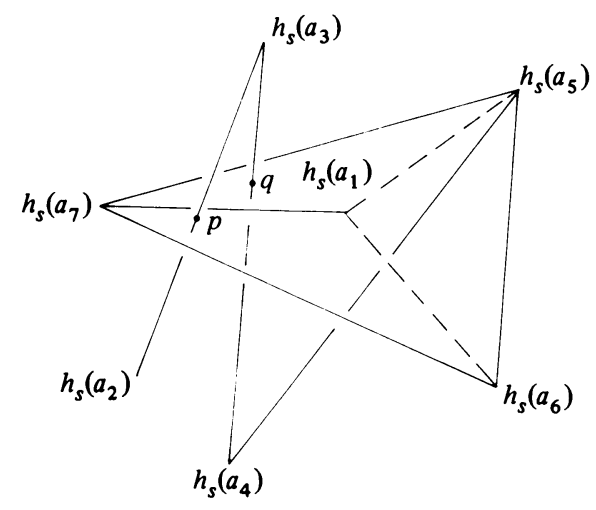

FIGURE 2.2

Let $p$ and $q$ be the points of intersection of the 2-simplex $h_{s}\left(a_{5}\right) h_{s}\left(a_{6}\right) h_{s}\left(a_{7}\right)$ with $h_{s}\left(a_{2} a_{3}\right)$ and $h_{s}\left(a_{3} a_{4}\right)$ respectively.

We claim that the 2-simplex $h_{s}\left(a_{4}\right) h_{s}\left(a_{5}\right) h_{s}\left(a_{7}\right)$ is pierced by neither $h_{s}\left(a_{2} a_{3}\right)$ nor $h_{s}\left(a_{1} a_{2}\right)$, thus contradicting Lemma 2.1. To see this fact note that the segment $h_{s}\left(a_{3} a_{4}\right)$ lies behind the plane $P_{174}$. Since $p$ is in front of $P_{174}, h_{s}\left(a_{2} a_{3}\right)$ must pierce $P_{174}$ above $P_{567}$. Hence the segment $p h_{s}\left(a_{2}\right)$ must lie in front of $P_{174}$. But the 2-simplex $h_{s}\left(a_{4}\right) h_{s}\left(a_{5}\right) h_{s}\left(a_{7}\right)$ lies behind $P_{174}$ since it is on the same side as $q$. Hence $h_{s}\left(a_{2} a_{3}\right)$ cannot intersect the 2-simplex $h_{s}\left(a_{4}\right) h_{s}$ $\left(a_{5}\right) h_{s}\left(a_{7}\right)$. Since $h_{s}\left(a_{2}\right)$ is in front of $P_{174}, h_{s}\left(a_{1} a_{2}\right)$ is also in front, hence $h_{s}\left(a_{1} a_{2}\right)$ cannot intersect it either. This proves that Example 2.1 has the properties claimed of it.

Since there is a PL homeomorphism $H$ of $E^{3}$ with compact support taking $f(C)$ to $g(C)$ which is linear on the simplexes in $f(C)$, Example 2.1 also serves to prove the following theorem.

THEOREM 2.1. There is a triangulation $T$ of a tetrahedron $\sigma$ and a linear homeomorphism $H: \sigma \rightarrow \sigma$ for which there is no linear isotopy $h_{t}: \sigma \rightarrow \sigma$ $(t \in[0,1])$ such that $h_{0}=\mathrm{id}$ and $h_{1}=H$.

This theorem also demonstrates a difference between the situation in $E^{2}$ and that in $E^{3}$. In fact, in $E^{2}$ the following theorem is true [2, Corollary 4.4]. If $T$ is a triangulation of a convex PL disk $P$ in $E^{2}$ and $H: P \rightarrow P$ is a linear homeomorphism of $(P, T)$ with $H \mid \mathrm{Bd} P=\mathrm{id}$, then there is a linear isotopy $h_{t}$ : 
$P \rightarrow P(t \in[0,1])$ so that $h_{0}=\mathrm{id}, h_{1}=H$, and for each $t, h_{t} \mid \mathrm{Bd} P=\mathrm{id}$.

Question 2.1. Let $g_{0}$ and $g_{1}$ be two linear embeddings of a simple closed curve $J$ into $E^{3}$ such that there is an orientation-preserving homeomorphism $h$ of $E^{3}$ with $h \circ g_{0}=g_{1}$. Then can the embedding $g_{0}$ be pushed to the embedding $g_{1}$ ? Suppose we assume that $g_{0}(J)$ is unknotted?

Question 2.2. Let $g$ be a linear embedding of a triangulated 2-sphere $S$. Can $g(S)$ be pushed onto the boundary of a tetrahedron?

3. Future work. In a future paper [6] the author will prove the following theorem.

TheOREM. Let $K$ be a finite complex and $f$ and $g$ be two PL embeddings of $K$ into $E^{3}$ so that there is an orientation-preserving homeomorphism $h$ of $E^{3}$ with $h \circ f=g$. Then there is a triangulation $T$ of $K$ and a linear isotopy (or push) $h_{t}$ : $(K, T) \rightarrow E^{3}(t \in[0,1])$ so that $h_{0}=f$ and $h_{1}=g$.

\section{REFERENCES}

1. R. H. Bing, An alternative proof that 3-manifolds can be triangulated, Ann. of Math. (2) 69 (1959), 37-65. MR 20 \# 7269.

2. R. H. Bing and M. Starbird, Linear isotopies in $E^{2}$, Trans. Amer. Math. Soc. (to appear).

3. S. S. Cairns, Isotopic deformations of geodesic complexes on the 2-sphere and on the plane, Ann. of Math. (2) 45 (1944), 207-217. MR 5, 273.

4. L. Euler, Opera postuma. I, Petropoli (1862), 464-496.

5. C. W. Ho, On certain homotopy properties of some spaces of linear and piecewise linear homeomorphisms. I,II, Trans. Amer. Math. Soc. 181 (1973), 213-243. MR 48 \# 1252.

6. M. Starbird, Linear isotopies in $E^{3}$, Proc. Amer. Math. Soc. (to appear).

Department of Mathematics, The University of Texas, Austin, TeXas 78712 